\title{
Measurement of the Phase Velocity of VLF Propagation in the Earth-Ionosphere Waveguide
}

\author{
F. K. Steele and C. J. Chilton
}

Contribution From the Central Radio Propagation Laboratory, National Bureau of Standards, Boulder, Colo.

(Received July 20, 1964)

\begin{abstract}
For short periods of time during June and July of 1963 the U.S. Navy transmitters located at Jim Creek, Wash. (NPG), and Balboa, Panama (NBA), simultaneously transmitted a frequency stabilized signal of $18 \mathrm{kc} / \mathrm{s}$. The phase and relative amplitude of these signals were monitored at Boulder, Colo.; College, Alaska; Maui, Hawaii; and Tucumán, Argentina. A semiempirical method by which the mean relative phase velocity can be calculated from the measurements of phase made at these four recording sites is demonstrated. The values of the mean relative phase velocity at $18 \mathrm{kc} / \mathrm{s}$ which are obtained for daytime and nighttime propagation conditions are respectively, $\left(V_{p} / c\right)_{d}=0.998$ and $\left(V_{p} / c\right)_{n}=0.995$.
\end{abstract}

\section{Introduction}

Early experimental work [Al'pert and Borodina, 1959; Jean, Taylor, and Wait, 1960] on the velocity of propagation of VLF radio waves utilizing the electromagnetic energy radiated from lightning discharges seemed to indicate for frequencies lower than about $20 \mathrm{kc} / \mathrm{s}$ that the phase velocity in the earthionosphere waveguide would be appreciably higher than the velocity of light, $c$ in vacuum. Taken together the above cited work gives essentially the same result for both day [Al'pert and Borodina, 1959] and night [Jean et al., 1960] propagation conditions. However, more recent VLF propagation measurements, obtained by means of the Radux-Omega navigation system [Casselman et al., 1959], have shown [Wait, 1961] that during nighttime propagation conditions the phase velocity is apparently lower than $c$ for frequencies greater than about 9 $\mathrm{kc} / \mathrm{s}$, and under daytime conditions lower than $c$ for frequencies greater than about $14 \mathrm{kc} / \mathrm{s}$.

Knowledge of the phase velocity in the earthionosphere waveguide is an important requirement for the proper interpretation of the phase variations observed in VLF propagation data and the observed systematic errors in phase-comparison types of VLF navigation aids. Thus, it is the purpose of this paper to present a method by which an estimate of the phase velocity can be obtained and, as an application of this method, to give the results obtained at a frequency of $18 \mathrm{kc} / \mathrm{s}$.

\section{Method of Measurement and Basic Theory}

For short periods of time during June and July of 1963 , the U.S. Navy transmitters NBA at Balboa, Panama, and NPG at Jim Creek, Wash., simultaneously transmitted phase stabilized signals at a frequency of $18 \mathrm{kc} / \mathrm{s}$ [F. M. Malone, private communication, 1963]. Measurements of the phase of the two signals were made using the transmitterreceiver network illustrated in figure 1 . The transmitter-receiver pairs required to make the measurements, from which a calculation of relative phase velocity can be obtained, is illustrated diagrammatically in figure 2. The symbols $R_{1}$ and $R_{2}$ represent the receivers, while $T_{1}$ represents the transmitter NPG at Jim Creek, Wash., and $T_{2}$ the transmitter NBA at Balboa, Panama. The propagation path lengths are $D_{1}, D_{2}, D_{3}$, and $D_{4}$. The received phases of NBA and NPG at $R_{1}$ (Boulder) and $R_{2}$ (Maui) are respectively $\left(\Phi_{1}, \Phi_{2}\right)$ and $\left(\Phi_{3}, \Phi_{4}\right)$. It was possible to measure the received phase of each signal at four monitoring sites: College, Alaska; Boulder, Colo.; Maui, Hawaii; and Tucumán, Argentina. These four receiving sites were combined to give six receiver pairs recording the $\mathrm{NBA}$ and $\mathrm{NPG}$ transmissions.

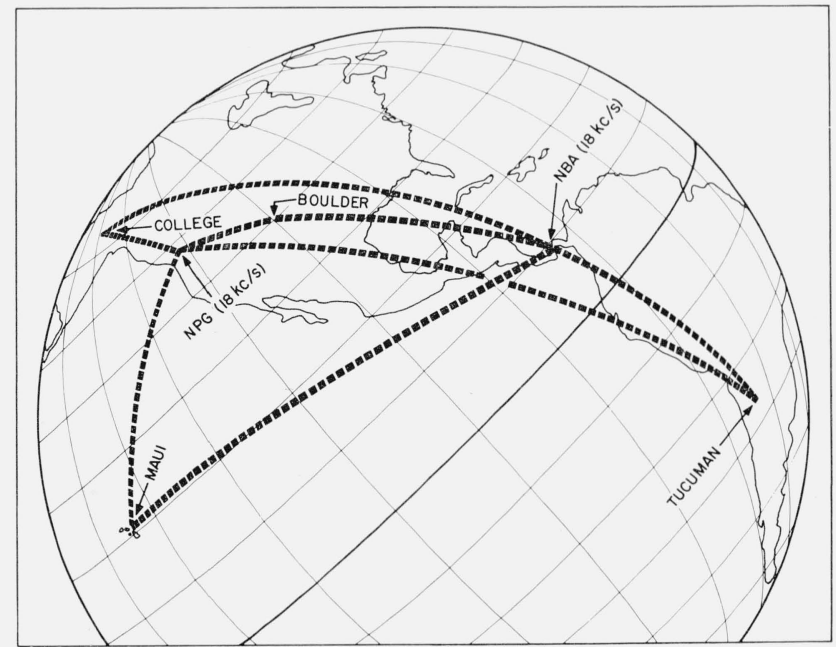

Figure 1. Geographic location of the VLF transmitter-receiver networks showing the propagation paths used in this study of relative phase velocity.

Each dash represents approximately 10 wavelengths of the $18 \mathrm{kc} / \mathrm{s}$ transmitter requency. 


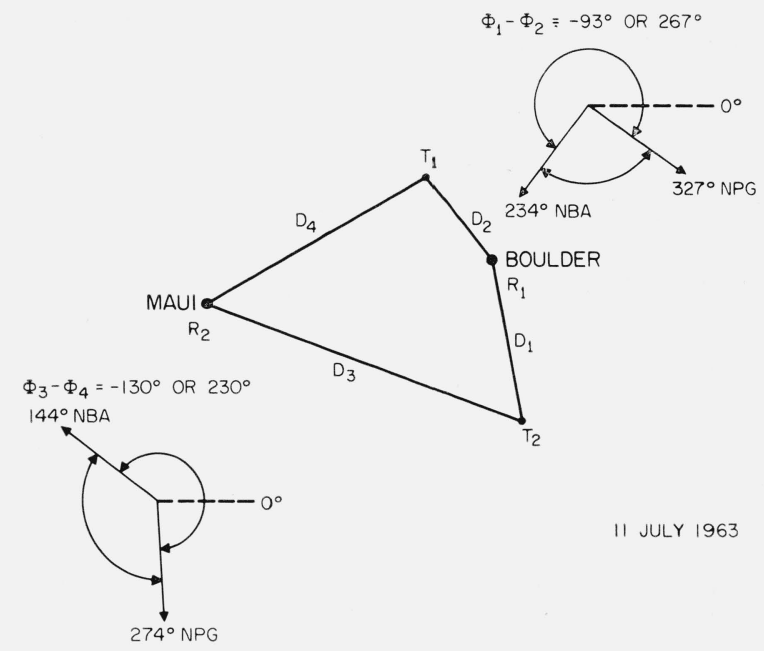

Figure 2. Diagrammatic representation of an individual transmitter-receiver network, upon which the calculation of a relative phase velocity is based.

The vector diagrams illustrate the cycle ambiguity arising at each receiving site in the calculation of the measured phase difference.

The data pairs used for this analysis are: MauiTucumán, College-Boulder, College-Maui, CollegeTucumán, Boulder-Maui, and Boulder-Tucumán. It will be shown in this paper that by assuming a uniform, isotropic, homogeneous ionosphere for the portion of the waveguide containing the two transmitters and four receiving sites, an effective value of the relative phase velocity, $V_{p} / c$, can be estimated from the data obtained from these pairs of recording sites.

A typical record, obtained at Boulder, Colo., is shown in figure 3 . In the time period from approximately 1800 U'T to $1900 \mathrm{U}$ 'T, both NBA and NPG transmitted on $18 \mathrm{kc} / \mathrm{s}$. The trace at the top of the record is the relative amplitude; the arrow on the right gives the direction of an amplitude increase. The trace at the bottom of the record is the phase; the

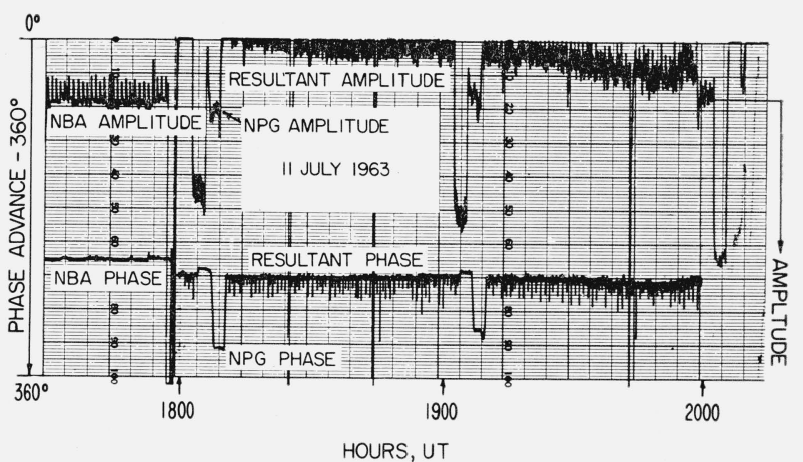

Figure 3. Copy of an original record of the phase and amplitude of the signals NBA (18 kc/s, Balboa, Panama) and NPG (18 kc/s, Jim Creek, Wash.) as recorded at Boulder, Colo.

arrow at the left gives the direction of a phase advance. The phase of NBA alone is indicated in the time period before $1800 \mathrm{U}$ T. The phase of NPG alone occurs in the time period from 1807 U'T to 1810 U'T when NBA is regularly not transmitting. Thus in these time periods at Boulder on 11 July 1963, the received phase $\left(\Phi_{1}\right)$ of $\mathrm{NBA}$ is $234^{\circ}$ and the received phase $\left(\Phi_{2}\right)$ of NPG is $327^{\circ}$. For purpose of illustration these values are shown in the vector diagram of figure 2. Similar records were taken at the other three recording sites, and the measured phase values of NPG and NBA from all of the usable records are tabulated (table 1). In table 1, the location of each recording site is given together with the date and measurement period in Universal Time. The data were separated according to solar controlled propagation conditions; i.e., either the propagation path was totally dark (all dark) or totally sunlit (all light). Each record gave a value of phase for NPG and NBA with respect to the receiver reference oscillator. Due to the electronic characteristics of the equipment, a possible cycle ambiguity (fig. 2 ) has to be taken into account when the phase difference between these two values is calculated.

Table 1. Measured VLF phase data

\begin{tabular}{|c|c|c|c|c|c|c|c|c|c|c|}
\hline \multirow{4}{*}{$\begin{array}{l}\text { Propagation } \\
\text { condition }\end{array}$} & \multirow{4}{*}{$\begin{array}{l}\text { Date } \\
1963\end{array}$} & \multirow{4}{*}{$\begin{array}{l}\text { Measure- } \\
\text { ment, } \\
\text { period } \\
\text { UT }\end{array}$} & \multicolumn{8}{|c|}{ Phase } \\
\hline & & & \multicolumn{8}{|c|}{ Location of recording site } \\
\hline & & & \multicolumn{2}{|c|}{ Boulder } & \multicolumn{2}{|c|}{ College } & \multicolumn{2}{|c|}{ Maui } & \multicolumn{2}{|c|}{ Tucumán } \\
\hline & & & NPG & NBA & NPG & NBA & NPG & NBA & NPG & NBA \\
\hline Path all dark...... & $\begin{array}{l}\text { 27 June } \\
\text { 28 June } \\
\text { 29 June } \\
\text { 30 June } \\
5 \text { July }\end{array}$ & $\begin{array}{l}0600-0800 \\
0600-0800 \\
0600-0800 \\
0600-0800 \\
0600-0800\end{array}$ & $\begin{array}{l}\operatorname{deg} \\
167 \\
274 \\
216 \\
162 \\
176\end{array}$ & $\begin{array}{r}\text { deg } \\
158 \\
291 \\
338 \\
320 \\
198\end{array}$ & $\begin{array}{l}\text { deg } \\
248 \\
241 \\
198\end{array}$ & $\begin{array}{l}\text { deg } \\
155 \\
173 \\
234\end{array}$ & $\begin{array}{r}\text { deg } \\
95 \\
36 \\
324\end{array}$ & $\begin{array}{r}\text { deg } \\
36 \\
353 \\
36\end{array}$ & $\begin{array}{r}\mathrm{deg} \\
223 \\
295 \\
192 \\
112 \\
263\end{array}$ & $\begin{array}{r}\text { deg } \\
97 \\
220 \\
135 \\
119 \\
144\end{array}$ \\
\hline Path all light...... & $\begin{array}{l}26 \text { June } \\
26 \text { June } \\
9 \text { July } \\
11 \text { July } \\
12 \text { July } \\
14 \text { July }\end{array}$ & $\begin{array}{l}1300-1500 \\
2100-2400 \\
1800-2100 \\
1800-2100 \\
1800-2100 \\
2100-2400\end{array}$ & $\begin{array}{l}270 \\
263 \\
327 \\
194\end{array}$ & \begin{tabular}{c}
342 \\
346 \\
\hdashline 234 \\
277
\end{tabular} & $\begin{array}{r}288 \\
288 \\
-\end{array}$ & \begin{tabular}{r}
65 \\
76 \\
\hdashline$-0 .-$ \\
\end{tabular} & $\begin{array}{l}238 \\
207 \\
274 \\
155 \\
111\end{array}$ & $\begin{array}{r}317 \\
112 \\
144 \\
162 \\
5\end{array}$ & $\begin{array}{r}329 \\
173 \\
252 \\
0 \\
25\end{array}$ & $\begin{array}{l}202 \\
230 \\
280 \\
220 \\
245\end{array}$ \\
\hline
\end{tabular}


In the analysis of the data we make use of the expression [Wait, 1961] that relates the phase velocity $\left(V_{p}\right)$ to the total phase path between the transmitter and receiver in the earth-ionosphere waveguide:

$$
\frac{\omega D}{V_{p}}=2 \pi M+\phi,
$$

where $\omega$ is the angular wave frequency, $D$ is the path distance, $M$ is the integral number of wavelengths in the propagation path, and $\phi$ is the additional fractional part of a cycle in the phase path. Thus we have for the propagation path system shown in figure 2 the four equations:

$$
\frac{\omega D_{i}}{V_{p}}=2 \pi M_{i}+\phi_{i}, \quad i=1,2,3,4 .
$$

Combining these four equations, and noting that the measured phase differences are given by $\Phi_{1}-\Phi_{2}=\phi_{1}-\phi_{2}+\gamma$ and $\Phi_{3}-\Phi_{4}=\phi_{3}-\phi_{4}+\gamma$, results in two expressions: one for the phase difference measured at receiver $R_{1}$,

$$
\left(\Phi_{1}-\Phi_{2}\right)=-2 \pi\left(M_{1}-M_{2}\right)+\left(\omega / V_{p}\right)\left(D_{1}-D_{2}\right)+\gamma,
$$

and one for the phase difference measured at receiver $R_{2}$,

$$
\left(\Phi_{3}-\Phi_{4}\right)=-2 \pi\left(M_{3}-M_{4}\right)+\left(\omega / V_{p}\right)\left(D_{3}-D_{4}\right)+\gamma,
$$

where $\gamma$ is the phase offset between the two transmitters. Equations (1) and (2) are then combined and solved for the phase velocity:

$$
\begin{aligned}
& V_{p}\left(\Phi_{i}, M_{i}, D_{i}\right) \\
& \quad=\frac{\omega\left[\left(D_{1}-D_{2}\right)-\left(D_{3}-D_{4}\right)\right]}{\left(\Phi_{1}-\Phi_{2}\right)-\left(\Phi_{3}-\Phi_{4}\right)+2 \pi\left[\left(M_{1}-M_{2}\right)-\left(M_{3}-M_{4}\right)\right]} .
\end{aligned}
$$

By letting $V_{p}=c$ we obtain an auxiliary equation:

$$
\begin{aligned}
c\left(\phi_{i}^{\prime}, M_{i}^{\prime}, D_{i}\right) & \\
& =\frac{\omega\left[\left(D_{1}-D_{2}\right)-\left(D_{3}-D_{4}\right)\right]}{\left(\phi_{1}^{\prime}-\phi_{2}^{\prime}\right)-\left(\phi_{3}^{\prime}-\phi_{4}^{\prime}\right)+2 \pi\left[\left(M_{1}^{\prime}-M_{2}^{\prime}\right)-\left(M_{3}^{\prime}-M_{4}^{\prime}\right)\right]},
\end{aligned}
$$

where $M_{i}^{\prime}$ and $\phi_{i}^{\prime}$ are respectively the calculated integral number of wavelengths, and additional fractional part of a cycle in the propagation path. These primed quantities $M_{i}^{\prime}$ and $\phi_{i}^{\prime}$ are calculated from the four equations:

$$
\frac{\omega D_{i}}{c}=2 \pi M_{i}^{\prime}+\phi_{i}^{\prime}, \quad i=1,2,3,4,
$$

where the geographic distances $D_{i}$ are computed, using the best available geographic coordinates, by the method of Lambert [1942] in which the earth is treated as an ellipsoid of revolution. Since Lambert's [1942] method gives a value of path distance which appears to differ from the true distance by less than one part in 200,000 [Sitterly and Pierce,
1944] even when the distance is nearly half a circumference, these values of $D_{i}$ are assumed to be within one wavelength (at $18 \mathrm{kc} / \mathrm{s}$, approximately $16 \mathrm{~km}$ ) of the true path length.

Finally, by taking the ratio $V_{p} / c$ we obtain the relation for the relative phase velocity in terms of measured and calculated phase path parameters:

$$
\frac{V_{p}}{c}=\frac{\left(\phi_{1}^{\prime}-\phi_{2}^{\prime}\right)-\left(\phi_{3}^{\prime}-\phi_{4}^{\prime}\right)+2 \pi\left[\left(M_{1}^{\prime}-M_{2}^{\prime}\right)-\left(M_{3}^{\prime}-M_{4}^{\prime}\right)\right]}{\left(\Phi_{1}-\Phi_{2}\right)-\left(\Phi_{3}-\Phi_{4}\right)+2 \pi\left[\left(M_{1}-M_{2}\right)-\left(M_{3}-M_{4}\right)\right]} .
$$

Using (3), the calculation of $V_{p} / c$ can be carried out by taking, as a starting point, the values of $M_{i}$ equal to the calculated $M_{i}^{\prime}$; then the $M_{i}$ are individually varied by integral numbers of wavelengths. Thus by a method of successive approximations a set of independent values is obtained for each receiver pair of measurements. Taken together these independent sets of values uniquely determine a value of $V_{p} / c$ which is common to each set. Then the average of these common values of relative phase velocity can be taken as the best estimate.

\section{Discussion and Analysis of the Data}

The eight VLF propagation paths, determined by the two transmitters and four receiving sites, give a total of six path systems for which calculations of the relative phase velocity $V_{p} / c$ can be carried out using (3). The cycle ambiguity illustrated in figure 2 and the variation of the $M_{i}$ by integral numbers result in four independent trial values of the ratio $V_{p} / c$ for each receiver pair. If any one of the four values is significant, this value should repeat itself at different times and in each set of calculations for each of the receiver pairs (Maui-Tucumán, CollegeBoulder, College-Maui, College-Tucumán, BoulderMaui, and Boulder-Tucumán). There were 11 periods consisting of 6 days and 5 nights when at least 2 of the 4 receiving sites obtained usable data. The data obtained during these 11 recording periods were analyzed in the manner outlined above and yielded a total of 140 calculated values of $V_{p} / c$. Sixty of these values were obtained for daytime propagation conditions, and 80 were obtained for nighttime conditions. The results of this analysis for nighttime conditions are shown in figure 4 . In this figure all of the calculated trial values of relative phase velocity for each measurement during nighttime propagation conditions are grouped according to receiver pairs as indicated. Thus each receiver pair shows columns of four possible values of the relative phase velocity where each column represents an independent experimental measurement for that receiver pair. There were four receiving sites which were combined to give six possible receiving pairs. That this combination constitutes an inherent check on the internal consistency of the data is made evident by the fact that in each set of 4 calculations (each column) only one value is found to be common to all 20 sets (columns). Although a considerable 


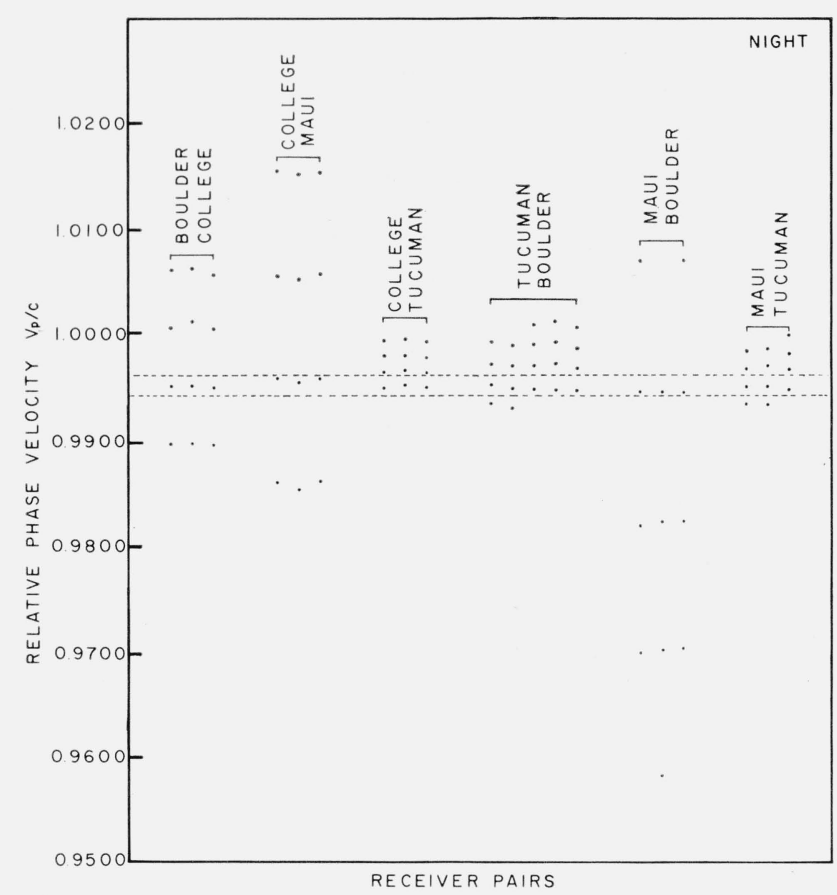

FIGURE 4. Calculated trial values of relative phase velocity for nighttime propagation conditions grouped according to receiver pairs.

Each column of four values represents an individual experimental measurement. The most probable value of $\left(V_{p} / c\right)_{n}$ is that which is common to each group and occurs between the dashed lines.

spread in each set of four trial values is evident, a value near 0.995 consistently appears in each grouping. These 20 values are set apart by dotted lines in figure 4 . The mean of these common values is 0.995 . For daytime conditions the analysis indicates a value near 0.998 as the common value of relative phase velocity (fig. 5). The mean of these daytime values is 0.998 .

A standard deviation of the mean for the above relative phase velocities was not given because (a) some of the data for the daytime case was rejected, and (b) only two in the six possible receiver pairs can be considered as being strictly independent. However, for the 20 values of the nighttime case the sample standard deviation is 0.0003 , giving 0.0001 as an estimate for the standard deviation of the mean even if the effective number of strictly independent values (of the 20) is as small as 5. In the daytime case, including the rejected 4 points to give a total of 15 values, the sample standard deviation is 0.0023 , giving 0.0009 as an estimate for the standard deviation of the mean where the effective number of strictly independent values (of the 15 ) is as small as 7 . However, if in the daytime case we use only the 11 accepted values lying between the dotted lines of figure 5 , the sample standard deviation is 0.0004 , giving 0.0002 as an estimate for the standard deviation of the mean even if the effective number of strictly independent values (of the 11) is as small as 7 . In this last case it is clear that this is a lower limit since it is possible that the

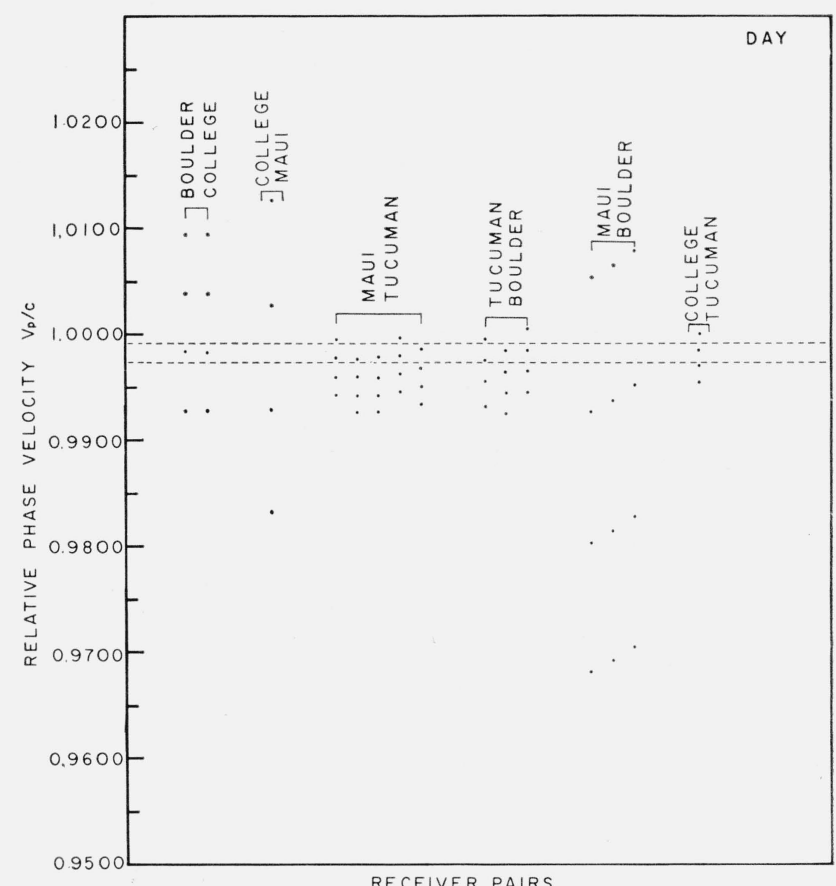

FigURE 5. Calculated trial values of relative phase velocity for daytime propagation conditions grouped according to receiver pairs.

Each column of four values represents an individual experimental measurement. The most probable value of $\left(V_{p} / c\right)_{d}$ is that which is common to each group and occurs between the dashed lines.

random errors, as evidenced by the four rejected values, may have exceeded the inherent ambiguities.

In the 20 sets of calculations for the nighttime relative phase velocity, a common value occurred 20 times. However, in the 15 sets of trial values for the daytime relative phase velocity, $\left(V_{p} / c\right)_{d}$, a common value occurred only 11 times. The fact that the common value did not occur as consistently in the daytime calculations as in the nighttime, is difficult to explain. The four values in the daytime calculations which did not fall into the general pattern arose from the pairing of the low magnetic latitude data from Maui, Hawaii with the higher magnetic latitude data from Boulder, Colo., and College, Alaska. This might point to a possible latitude variation in the height of the daytime ionosphere [Chilton et al., 1964]. Nevertheless, other parameters such as the direction of propagation may play an important role and should therefore be considered. However, the limited amount of data presently available is not sufficient for the resolution of this problem.

The above values of experimentally deduced relative phase velocities are in good agreement with the theory of the first order mode for VLF propagation in a curved earth-ionosphere waveguide [Wait, 1963]. Specifically, theoretical calculations by Spies and Wait [1961] show that for a reasonable model of the ionosphere and for a perfectly conducting ground the relative phase velocities obtained above correspond to effective heights of reflection of $70 \mathrm{~km}$ during the day, and $95 \mathrm{~km}$ at night. Since the propagation 
paths used in this study cover a large variation in latitude and longitude these values of effective height should only be taken as an approximation. This is emphasized by the fact that the difference in calculated diurnal height change between the NBABoulder path and the NBA-Tacumán path is on the order of $5 \mathrm{~km}$ [Chilton et al., 1964] even though the two paths are essentially the same length.

It is difficult to compare the above phase velocities with the earlier broadband experimental results [Jean et al., 1960; Al'pert and Borodina, 1959] due to the fact, not specifically stated in either paper, that in their analysis the phase velocities at higher frequencies were assumed and taken to be equal to the velocity of light. On the other hand, our values at $18 \mathrm{kc} / \mathrm{s}$ compare favorably with extrapolated values from the later work [Wait, 1961] done in the frequency range from $8 \mathrm{kc} / \mathrm{s}$ to $16 \mathrm{kc} / \mathrm{s}$.

\section{Conclusions}

This paper shows that for a frequency of $18 \mathrm{kc} / \mathrm{s}$ and for that portion of the earth-ionosphere waveguide covered by the transmitter-receiver network shown in figure 1 , the mean effective relative phase velocities are $\left(V_{p} / c\right)_{d}=0.998$ by day and $\left(V_{p} / c\right)_{n}=$ 0.995 by night.

Although the experimental technique and method of analysis of the data are relatively uncomplicated, they yield results which are strongly dependent on having enough data to minimize the ambiguities and experimental errors which are inherent in the measurements. There is some evidence in the observations reported here which might suggest that the daytime phase velocity depends on path latitude and possibly direction of propagation. However, further observations on more paths and at different frequencies are required to verify this conclusion.
The authors thank D. D. Crombie for his encouragement and suggestions. The observations at College, Alaska, were made under the supervision of Dr. H. F. Bates at the Geophysical Institute, University of Alaska. Those at Tucumán, Argentina, were made under the supervision of Sandro M. Radicella of the Instituto de Electrotecnia, Universidad Nacional de Tucumán. The observations at Maui, Hawaii, were made by S. Katahara, while those at Boulder, Colo., were under the supervision of A. H. Diede.

\section{References}

Al'pert, Ia. L., and S. V. Borodina (1959), On the velocity of propagation of electromagnetic waves, Radiotekh. i Elektron 3, 195.

Casselman, C. J., D. P. Heritage, and M. L. Tibbals (May 1959), VLF propagation measurements for the raduxomega navigation system, Part 1, Proc. IRE 4\%, No. 5, 829-839.

Chilton, C. J., A. H. Diede, and Sandro M. Radicella (Apr. 1, 1964), Transequatorial reception of a very-low-frequency transmission, J. Geophys. Res. 69, No. 7, 1319-1328.

Jean, A. Glenn, William L. Taylor, and James R. Wait (March 1960), VLF phase characteristics deduced from atmospheric wave forms, J. Geophys. Res., 65, No. 3, 907-912.

Lambert, Walter D. (May 15, 1942), The distance between two widely separated points on the surface of the earth, J. Wash. Acad. Sci. 32, No. 5, 125-130.

Sitterly, B. W., and J. A. Pierce (July 1944), Simple computation of distance on the earth's surface. Radiation Laboratory, Massachusetts Institute of Technology, Report 582 .

Spies, K. P., and J. R. Wait (July 17, 1961), Mode calculations for VLF propagation in the earth-ionosphere waveguide, NBS Tech. Note 114.

Wait, J. R. (June 1961), A comparison between theoretical and experimental data on the phase velocity of VLF radio waves, Proc. IRE 49, No. 6, 1089-90.

Wait, J. R. (1962), Electromagnetic Waves in Stratified Media (Pergamon Press, New York), Ch. VII, 196-225.

(Paper 68D12-431) 


\title{
Comments on a Paper "Measurement of the Phase Velocity of VLF Propagation in the Earth Ionosphere Waveguide" by F. K. Steele and C. J. Chilton
}

\author{
Howard F. Bates \\ Geophysical Institute, University of Alaska, College, Alaska
}

(Received March 4, 1965)

Several comments are in order on the phase velocities derived by Steele and Chilton [1964].

1. The NPG and NBA to College paths were not totally dark, as claimed, between 0600 and 0800 UT in late June and early July. In fact, College was actually in sunlight at those times. By the end of their observation period on July 13 , only roughly 25 percent of the $D$ region at 0600 was dark on the NPGCollege path, and 75 percent on the NBA-College path; by 0800 the amounts that were dark increased to about 60 and 90 percent, respectively. The inclusion of the College data in their computation of the nighttime phase velocities therefore seems questionable.

2. Nighttime second mode propagation effects cannot be ignored on any of the paths discussed, particularly the NPG to Boulder and College paths. Both of these paths are so short that the second mode may well predominate at night [W att and Croghan, 1964]. In addition, the observed nighttime phase and amplitude effects observed on various longer VLF paths, including some of the paths Steele and Chilton discuss, can only be consistently explained by a mode-interference model in which the first and second modes are of roughly comparable amplitude on 4000 to $5000 \mathrm{~km}$ paths [Bates and Albee, 1965]. Crombie's [1964] apparently correct explanation of the sunrise fading effect relies upon a strong nighttime second mode propagating to distances well over $7500 \mathrm{~km}$; quantitative bounds on the second mode amplitude can be obtained from his model.

The equations used by Steele and Chilton are valid only for single-mode propagation. Therefore, the nighttime phase velocities they found should be recomputed with phase interference effects included.

3 . It is not specifically stated whether the most probable values of phase velocity they give correspond to the same day and night values of the $M_{i}$ for each path. This would appear to be a test for consistency. Also, the values of $M_{i}$ found for each path would seem to be an important parameter of VLF propagation.

\section{References}

Bates, H. F., and P. R. Albee (1965), General VLF phase variations observed at College, Alaska, J. Geophys. Res. 70, 2187-2208.

Crombie, D. D. (1964), Periodic fading of VLF signals received over long paths during sunrise and sunset, Radio Sci. J. Res NBS 68D, No. 1, 27-34.

Steele, F. K. and C. J. Chilton (1964), Measurement of the phase velocity of VLF propagation in the earth-ionosphere waveguide, Radio Sci. J. Res. NBS 68D, No. 12, 1269-1273.

Watt, A. D. and R. D. Croghan (1964), Comparison of observed VLF attenuation rates and excitation factors with theory, Radio Sci. J. Res. NBS 68D, No. 1, 1-10.

(Paper 69D8-551) 


\title{
Reply to H. F. Bates', Comments
}

\author{
F. K. Steele and C. J. Chilton \\ National Bureau of Standards, Boulder, Colo.
}

(Received March 23, 1965)

In the following we reply to Bates' comments in the order of their presentation.

1. Our calculations show that on 28 June 1963 the average solar zenith angle $(\chi)$ over the NPG-College path varies from $90^{\circ}$ to $94^{\circ}$ during the measurement period (0600 to $0800 \mathrm{UT}$ ), and for the NBA-College path varies from $90^{\circ}$ to $94^{\circ}$ during the measurement is from $94^{\circ}$ to $96^{\circ}$. On the basis of these calculations we have called the path totally dark when the average zenith angle is greater than $90^{\circ}$. However, we agree with Bates that portions of the two College paths were weakly illuminated, but the paths can be considered to be more nearly representative of nighttime conditions than of daytime conditions. Nevertheless, the inclusion of the College data in the computations of the nighttime phase velocities might seem questionable. Let us assume for the moment that the College, Alaska, data are marginal. Eliminating these data from the analysis does not change the result, i.e., the calculated average value of the phase velocity remains the same.

2. Whether or not the nighttime second mode propagation effects can be ignored depends on the relative attenuation $(\alpha)$ of the second-order mode compared to the first-order mode, and the magnitude of the excitation factor $(\Lambda)$ for the second-order mode compared to the first-order mode. For frequencies greater than $20 \mathrm{kc} / \mathrm{s}$ both theory [Wait and Spies, 1964] and experiment [Watt and Croghan, 1964] indicate that there may very well be an appreciable second-order mode effect. However, for frequencies less than $20 \mathrm{kc} / \mathrm{s}$ both theory [Wait and Spies, 1964] and experiment [Wait, 1961; Watt and Croghan, 1964] indicate that the effect of the second-order mode is small if not negligible at distances greater than 3000 $\mathrm{km}$. In point of fact, our measurements were made at $18 \mathrm{kc} / \mathrm{s}$, and all but two of the eight propagation paths had distances greater than $4000 \mathrm{~km}$. The results obtained using the two shorter paths do not appear to be inconsistent with those obtained from the rest of the data. In regard to Bates' interpretation of Crombie's [1964] explanation of the sunrise fading effects, this theory does not necessarily require a "strong nighttime second mode propagating to dis- tances well over $7500 \mathrm{~km}$," but the observed results could possibly be due to additive effects of mode conversion in a distributed sunrise region [Crombie, 1965 private communications]. In addition, it should be remembered that Crombie's published results refer to paths where propagation was to the east, whereas the paths in the paper under discussion were mainly for propagation to the west, in which case attenuation of the second-order mode is considerably increased relative to that of the first-order mode.

3 . The $M_{i}$ are the integral number of wavelengths in the respective propagation paths. In the equation (3), used to calculate the relative phase velocity, they are additively summed: $\left[\left(M_{1}-M_{2}\right)-\left(M_{3}-M_{4}\right)\right]$. We did not attempt to evaluate an absolute $M_{i}$ for each path, because (a) the $M_{i}$ are not initially known or measured but are obtained as a first approximation by calculation assuming the velocity of light as the phase velocity, and (b) there is a cycle ambiguity, inherent in each measuring equipment (see fig. 2), which could amount to a wavelength. It was therefore necessary to vary the estimated $M_{i}$, and thus the sum, by integral numbers of wavelengths. However, we agree with Bates that the $M_{i}$ are important parameters in VLF propagation and, if one assumes our values of phase velocity at $18 \mathrm{kc} / \mathrm{s}$, they can be calculated for each path by evaluating the expression [Wait, 1961] that relates the phase velocity to the total phase path between the transmitter and receiver in the earth-ionosphere waveguide.

\section{References}

Crombie, D. D. (1964), Periodic fading of VLF signals received over long paths during sunrise and sunset, Radio Sci. J. Res. NBS 68D, No. 1, 27-34.

Wait, J. R. (1961), A comparison between theoretical and experimental data on the phase velocity of VLF radio waves, Proc. IRE 49, No. 6, 1089-90.

Wait, J. R., and K. P. Spies (1964), Characteristics of the earthionosphere waveguide for VLF radio waves, NBS Tech Note No. 300.

Watt, A. D., and R. D. Croghan (1964), Comparison of observed VLF attenuation rates and excitation factors with theory, Radio Sci. J. Res. NBS 68D, No. 1, 1-10.

(Paper 69D8-552) 